\title{
Sunglass filter transmission and its operational effect in solar protection for civilian pilots
}

Adrian C Chorley ${ }^{1}$, Andrey Lyachev², Michael P Higlett ${ }^{2}$, Marina Khazova², Martin J Benwell ${ }^{3}$, Bruce JW Evans ${ }^{3,4}$

1. UK Civil Aviation Authority

2. Public Health England UK

3. London South Bank University, UK

4. Institute of Optometry, $U K$

Corresponding Author:

Dr Adrian Chorley BSc (Hons) MSc PhD FCOptom

UK CAA Medical Department

Safety and Airspace Regulation Group

Aviation House

Gatwick Airport South

Sussex RH6 OYR

adrian.chorley@.caa.co.uk

Tel: 01293573637 


\section{Co-author details:}

Dr Andrey Lyachev PhD, MPhil

Laser and Optical Radiation Dosimetry Group, Public Health England, Chilton, UK

Dr Michael P Higlett PhD, MPhys

Laser and Optical Radiation Dosimetry Group, Public Health England, Chilton, UK

Dr Marina Khazova PhD

Laser and Optical Radiation Dosimetry Group, Public Health England, Chilton, UK

Dr Martin J Benwell PhD BSc (Hons)

Associate Professor, School of Health and Social Care, London South Bank University, UK

Professor Bruce JW Evans BSc (Hons) PhD FCOptom

Director of Research, Institute of Optometry, UK

Visiting Professor, School of Health and Social Care, London South Bank University, UK

Suggested short title: Sunglass protection in civilian pilots

Word Count: Abstract: 249

Main Text: 4311

No. of References: $\quad 39$

No. of Tables: $\quad 2$

No. of Figures: $\quad 2$ 


\section{Abstract}

INTRODUCTION: The ocular effects of excess solar radiation exposure are well documented. Recent evidence suggests that ocular ultraviolet radiation (UVR) exposure to professional pilots may fall outside international guideline limits unless eye protection is used. Non-prescription sunglasses should be manufactured to meet either international or national standards. The mean increase in UVR and blue light hazards at altitude has been quantified and the aim of this research was to assess the effectiveness of typical pilot sunglasses in reducing UVR and blue light hazard exposure in flight. METHOD: A series of sunglass filter transmittance measurements were taken from personal sunglasses $(n=20)$ used by pilots together with a series of new sunglasses $(n=18)$. RESULTS: All nonprescription sunglasses measured conformed to international standards for UVR transmittance and offered sufficient UVR protection for pilots. There was no difference between right and left lenses or between new and used sunglasses. All sunglasses offered sufficient attenuation relevant to counter the mean increase in blue light exposure pilots experienced at altitude; however, the used sunglasses with scratched lenses were least effective. One pair of prescription sunglasses offered insufficient UVR attenuation for some flights but would have met requirements of international and national standards for UVA transmittance. This was likely due to insufficient UVR blocking properties of the lens material. CONCLUSIONS: Lenses manufactured to minimally comply with standards for UVR transmittance could result in excess UVR exposure to a pilot based on in-flight irradiance data; an additional requirement of less than $10 \%$ transmittance at $380 \mathrm{~nm}$ is recommended.

Keywords: airline, pilots, sunlight, sunglasses, UV, blue light hazard 


\section{Introduction}

Ultraviolet radiation (UVR) is typically defined within the waveband 100 to $400 \mathrm{~nm}$ (28) and is subdivided into UV-C (100 - $280 \mathrm{~nm})$ which is absorbed by stratospheric oxygen, UV-B (280 - $315 \mathrm{~nm})$, much of which is absorbed by atmospheric ozone and UV-A $(315-400 \mathrm{~nm})$ which is the least energetic UVR waveband and which constitutes around 95\% of total terrestrial UVR (32).

It is known that UVR increases with altitude by $10-12 \%$ every $1,000 \mathrm{~m}$ (4). This translates to a two to three times increase in UVR between sea level and an airline cruise altitude of 35,000 ft (6). Factors influencing ocular exposure include the position of the sun in relation to the aircraft, reflection of radiation from surfaces below the aircraft such as snow or cloud top, the filtering effect of the ozone layer, altitude, the transmission properties of the cockpit windshield and the pilot's use of eye protection such as sunglasses.

UVR below $280 \mathrm{~nm}$ is absorbed by the cornea. At increasing wavelengths, an increasing proportion of incident radiation is transmitted through the cornea and absorbed within the aqueous, lens or vitreous. At $380 \mathrm{~nm}, 80 \%$ of radiation is transmitted through the cornea (38). The lens absorption is strongest within the 340-380nm range and lenticular UVR transmission decreases steadily with age (38).

UVR exposure is associated with cataract formation $(12,16,30)$; however, evidence of increased prevalence of cataracts in pilots is limited $(6)$. There is some evidence $(21,37)$ of a relationship between UV-A exposure and melanocytic skin cancers which, if present on the eyelids, can have significant effect to comfort or vision. Evidence also points to a higher incidence of melanoma in aircrew (33). Exposure to elevated levels of short wavelength visible light has been associated with permanent damage to retinal photoreceptors $(1,35,39)$. This is known as the blue light hazard which has its peak efficacy around 440nm for an eye with a natural lens (24). Retinal damage has been 
attributed to short exposure to high intensity artificial light sources; there is also evidence that long term exposure to solar radiation may cause similar clinical signs as seen in short exposures which mimic the clinical changes seen in age related macular degeneration (AMD) (20). However, large scale population studies of AMD and sunlight exposure are inconclusive $(13,36)$ and there is no available evidence as to the prevalence of AMD in the professional pilot population (6).

It is known that pilots are subject to bright light conditions during flight and there are a number of sources $(11,18)$ which offer guidance to pilots on sunglass selection; however, these recommendations are not underpinned by evidence of actual in-flight levels of irradiance or of increased radiation related ocular pathology amongst pilots (6). Although it is recognised that there is a wide population variation in sunlight tolerance and there is likely to be a proportion of the pilot population who do not feel the need to use sunglasses in flight for visual comfort, a number of barriers to sunglass use in pilots have been identified (7) which hinder the successful use of sunglasses in a cohort who would otherwise wish to use them more.

There are two important benefits of wearing sunglasses: to provide the user ocular comfort in bright light conditions and to protect from harmful radiation. Non-prescription sunglasses are manufactured to comply with international or national standards $(2,3,26)$ which lay down minimum criteria for sunglass manufacture. These include lens impact resistance, luminous transmittance and solar UVR transmittance. Studies have reported on the compliance of sunglasses to sunglass filter standards $(14,15)$. Although a number of sunglasses measured in these studies were found to be non-compliant (15), this was generally due to factors other than UVR attenuation.

There are a number of differences between the national and international sunglass filter standards, one of which is the definition of the UV-A waveband for the required tests. The International Organisation for Standardization (ISO) and the American National Standards Institute (ANSI) define UV-A in the waveband $315-380 \mathrm{~nm}$ whereas the Australian/New Zealand Standard (AS/NZS), which also require mandatory testing, define UV-A in the waveband $315-400 \mathrm{~nm}$. Furthermore, UV-A 
transmittance in ISO 12312-1 standard is defined as a spectrally weighted value using spectral effectiveness function, $S(\lambda)(21)$, whereas the UV-A Exposure Limit Values (ELVs) recommended by the International Commission on Non-Ionizing Radiation Protection (ICNIRP) to protect the eye from UVR-induced cataract are given in un-weighted radiant exposure together with spectrally weighted ELVs for the whole spectral range of $180-400 \mathrm{~nm}(21,23)$. ANSI Z80.3 - 2010 define UV-A transmittance as the mean (unweighted) transmittance in the spectral range $315-380 \mathrm{~nm}$.

Significant ocular exposures to UV-A have been measured during flight $(8,10)$. This is present even with use of standard aircraft protection systems such as visors; however, the findings relate to an unprotected eye and exposure to UV-A will be reduced by the use of sunglasses. The level of UV-A irradiance during flight was largely dependent on windshield type. Recent research revealed aircraft windshields that appear to be identical can be placed in two distinct categories in terms of the UV-A absorption (10,31). Those which effectively block UV-A radiation up to the end of the UV-A waveband $(400 \mathrm{~nm})$ and those which effectively blocked up to around $340-350 \mathrm{~nm}$ but transmit UVA radiation beyond this point. The former windshield type has been found not to cause an ocular exposure to the unprotected eye beyond international guideline limits, regardless of flight duration or relative position of the sun to the pilot. The latter type of windshield results in unprotected ocular exposure to UV-A to exceed ICNIRP limits $(21,23)$ on all flights and, potentially, over-exposure within one hour of flight (10). No significant UV-B radiant exposures to the pilot have been found during flight $(10,17)$. The difference in transmittance between the two windshield types is likely to be due to the presence of a plastic material such as acrylic or polycarbonate either as a component of a multi-plied windshield structure (as found in airline aircraft types) or as a purely plastic material as typically found in general aviation aircraft. Additional occupational UVR exposure, unfiltered by a windshield may occur during any outside pre-flight activities such as aircraft walkaround. There is evidence that pilots use sunglasses less during this activity than during cruise flight (9). 
There are no national or international sunglass standards for blue light hazard protection as evidence suggests that solar exposure during normal day to day activities would not cause retinal damage (26); however, the long term effects are less well known. ISO and ANSI recognise that direct viewing of the sun is hazardous and the Standards include a definition of solar blue light transmittance of filters but do not contain a mandatory requirement for this characteristic. There are international guideline limits on ocular exposure to the blue light hazard (24). Whilst sunglasses would reduce exposure to blue light, it is possible that a strongly attenuating blue light filter may impact, to a degree, the user's color perception (29) and may also interfere with circadian entrainment regulated by melatonin which peaks around $450-475 \mathrm{~nm}(5)$.

The increase in blue light exposure at altitude has been quantified using the same protocol as for UV-A irradiance measurements (8) and although a large variation between ground and altitude exposure was measured, there was a mean increase of 4.1 times at altitude. Exposure to blue light fell well within ICNIRP limits for exposure to the unprotected eye; however, the accumulative effect of this increase in exposure over a flying career is unknown.

The aim of this study was to assess the effectiveness of UVR and blue light protection afforded by sunglasses typically used by pilots occupationally (9).

\section{Methods}

\section{Subjects}

A UK airline was approached and gave approval for researchers to undertake transmission measurements from their pilot employees' sunglasses subject to individual consent. All subjects were asked to loan their personal sunglasses for measurement whilst they were present in the airline's crew room at Gatwick Airport, UK where the measuring equipment was set up. Following measurement, the sunglasses were returned to the pilot. Data collection took place on 8 July 2013. 
The study had research ethics approval from London South Bank University and the Institute of Optometry, London.

\section{Equipment}

Measurements were carried out using an Ocean Optics HR4000 miniature CCD array spectrometer (Ocean Optics Inc, Dunedin, Florida, USA) connected to 74-UV collimating lens (Ocean Optics Inc, Dunedin, Florida, USA) by metal sleeved QP600-2-UV/BX (Ocean Optics Inc, Dunedin, Florida, USA) 2 metre fibre optic cable. The light source was an Ocean Optics DT-MINI-2-GS (Ocean Optics Inc, Dunedin, Florida, USA) which combined deuterium and tungsten-halogen lamps to generate a continuous spectral power distribution throughout the UVR and visible range. The light source had a shutter switch allowing dark measurements to be taken without turning the lamp off. The lamp was connected to a second collimating lens by a QP600-2-UV/BX 2 metre fibre optic cable. The lenses of light source and input optics of spectrometer were secured to an Ocean Optics Adjustable Collimating Lens Holder (Ocean Optics Inc, Dunedin, Florida, USA) which allowed the pair of collimating lenses to be installed co-axially at a chosen height. The distance between the collimating lenses was set to a minimum sufficient to allow placement of a sunglass filter between the lenses.

ISO 12312-1, 2013 transmittance requirements are based on sample measurement using a CIE Standard Illuminant D65 (25). This is important where a single value is obtained using a broad-band meter between $380-780 \mathrm{~nm}$. Where spectral transmittance is measured, as in this research, luminous transmittance is calculated taking account of the relative sensitivity of the human eye $V(\lambda)$ and the spectral energy distribution of $D 65 S_{D 65}(\lambda)(27)$. Therefore, provided the source has sufficient output within the required spectral range, the calculations with reference to ISO are unaffected by the use of a different light source.

\section{Procedure}


Details were taken from each pair of sunglasses including make and model (usually available on the inside surface of one of the spectacle frame sides), details of whether the lenses were known to be photochromic, prescription, polarised or graduated (gradient) tints and predominant tint color based on visual inspection. The sunglasses were also assessed for lens degradation (such as scratches) and cleanliness.

Each sunglass lens was placed at the estimated geometric centre at a normal plane to the collimated beam from the illumination source with the frame in a horizontal position. Spectral measurements together with dark measurements were taken from left and right lenses of each pair of sunglasses. Reference spectra from the source together with dark measurements were taken at the start and end of the data collection session. This measurement method is broadly equivalent to simultaneous acquisition of reference and transmitted beams by dual double beam spectrophotometers often used for transmission measurements.

Where the sunglasses lenses were seen to be dirty, a second set of measurements were taken after cleaning the lenses with a lens cleaning cloth. To determine whether the HR4000 was affected by polarised radiation, sunglass filters known to be polarised were additionally measured with the sunglasses rotated so that the right and left lenses were as close to a vertical plane as practical. Graduated tinted lenses were measured at three vertical points on each lens. These were at the top for the maximum tint, at the bottom of the lens for the minimum tint and at the estimated geometric centre. Photochromic lenses were measured but were eliminated from analysis due to the inability to control for their level of activation.

A community based optometry practice was approached and agreed to allow transmittance measurements for a series of new sunglasses. This was carried out on 11 July 2013 and after the crew room data collection when equivalent models of the most prevalent sunglass types measured in the crew room could be assessed. Where possible, the same sunglass model was used. The equipment and protocol for measurement were the same as for the used sunglass measurements. 


\section{Data analysis}

Lamp reference and sunglass filter spectral data were used to calculate:

- Spectral transmittance in $1 \mathrm{~nm}$ step calculated by subtracting the dark reading from each spectral measurement and using integration time to calculate the counts per second (cps) value for each wavelength step. These were expressed as a percentage value of the equivalent wavelength step cps values of the source without the filter in place.

- Luminous transmittance $\tau_{v}$, mean UV-A transmittance (as defined by ANSI Z80.3 standard) and spectrally weighted UV-A transmittance $\tau_{\mathrm{UVA}}$ (as defined by ISO 12311 standard) in order to classify filter category;

- Solar blue light transmittance as defined by ISO 12311 standard

- Wavelength threshold for 1, 2 and 5\% UV-A transmittance. These were found to be useful reference threshold points for grouping the data based on the range of transmittance of the sample of sunglasses measured.

Sunglass spectral transmittance data of selected prescription and all non-prescription sunglasses were used for the assessment of ocular protection from UV-A and blue light weightedirradiance measured during flight.

\section{Results}

Details of the sunglasses assessed are shown in Table I. A total of 20 used non-prescription pilot sunglasses were measured, consisting of 15 pairs with a uniform tint and 5 pairs with a graduated tint. A total of 18 new sunglasses were measured ( 12 uniform and 6 graduated tints).

[Table I here]

A further 12 pairs of used sunglasses were measured but excluded from main data analysis. Six were prescription sunglasses and a further five (Serengeti) were known to be photochromic. Additionally, 
data from a pair of Oakley sunglasses showed a graduated tint and a transmission curve similar to a photochromic lens. Oakley product information revealed that the sunglasses were not manufactured with a graduated tint and it was suspected that photochromic lenses had been glazed into this particular frame at some time after initial purchase; therefore, these data were excluded.

The differences in left-right inter-lens transmittance for measured sunglasses were insignificant. For analysis, the mean transmittance was used. Transmittance measurements were unaffected by lens polarisation. Transmission measurements taken before and after cleaning of marked or dirty filters showed no measurable difference in the UV-A range.

Five categories of sunglass filters are defined by ISO 12312-1 (26). Two of these (filter categories 2 and 3) apply for general purpose sunglasses. The luminous transmittance $\tau_{v}$ of the category 2 filter should be in the range between 18 - 43\% and for category 3 filters between 8 - 18\%; maximum value of solar UV-A transmittance should not exceed $0.5 \tau_{v}$. With the exception of three pairs, all measured uniform tint sunglasses fell into category 3; two pairs into category 2 and a single pair into category 4 (classified 'very dark special purpose sunglasses') with a luminous transmittance of $4.4 \%$. Sunglasses with graduated tints, when assessed at the geometric centre of the lens, fell into category 2, with the exception of one pair which had category 3 filters. Weighted UV-A transmittance of all non-prescription uniform and graduated tint lenses did not exceed $0.5 \tau_{v}$.

ANSI Z80.3: 2010 defines four shades of non-prescription sunglasses. The luminous transmittance $\tau_{v}$ of a General Purpose lens or shields should be in the range between $8-40 \%$ and with the single exception all measured uniform tint sunglasses fell into this category; a single pair (classified as category 4 filters according to ISO 12312-1) would be considered as Special Purpose very dark lens. Maximum value of UV-A transmittance should not exceed $0.5 \tau_{v}$ for high and prolonged exposure (2); UV-A transmittance of all sunglasses complied with the requirements of ANSI Z80.3: 2010. 
The maximum UV-A spectral transmittance was below $5 \%$ for the majority of uniform and graduated sunglasses as illustrated in Fig. 1. It should be noted that UV-A transmittance in this analysis is a spectral transmittance in the range $315-400 \mathrm{~nm}$ and not spectrally weighted as used in the ISO standard.

[Fig. 1. Here]

The transmittance of all new and used sunglasses at $380 \mathrm{~nm}$ ranged between $0.1-0.6 \%$ (mean $0.3 \%$ for uniform and $0.4 \%$ for graduated tints) providing very high UV-A attenuation. At $400 \mathrm{~nm}$, transmittance ranged between $0.1-7.0 \%$ (mean $1.1 \%$ for uniform and $1.3 \%$ for graduated tints) Three pairs of sunglasses (two used uniform tints and one new graduated tint) demonstrated a transmittance over $5 \%$ at $400 \mathrm{~nm}$. These values were $7.0 \%, 5.3 \%$ and $6.1 \%$ respectively. The wavelength threshold at which the transmittance exceeds $1 \%, 2 \%$ and $5 \%$ for uniform and graduated sunglasses was assessed. The majority of sunglasses assessed (13 used and 15 new) did not exceed a transmittance of $1 \%$ at $400 \mathrm{~nm}$; three new and seven used sunglasses exceeded $1 \%$ transmittance at a wavelength below $400 \mathrm{~nm}$; no sunglasses exceeded a $1 \%$ transmittance below $386 \mathrm{~nm}$.

For the assessment of reduction of blue light exposure, solar blue light weighted transmittance (21) was analysed. Blue light transmittance varied between $0.6 \%$ and $16 \%$, with the majority within the range 10 -13\%; all measured sunglasses provided sufficient blue light attenuation to counteract increase of irradiance level at altitude. Highest transmittance occurred in sunglasses recorded as having scratched lenses.

\section{Discussion}

Out of the used non-prescription pilot sunglasses presented for measurement, the most prevalent were RayBan (36\%), Oakley (21\%) and Serengeti (21\%). Although, the latter sunglass type were 
excluded from analysis, a recent study investigating sunglass use in pilots (9) also found these to be the three most prevalent types reported as used in flight (32\%, 19\% and 9\% respectively).

All non-prescription uniform and graduated tinted sunglasses measured showed good UV-A attenuation within $0.6 \%$ to $380 \mathrm{~nm}$. Between $380-400 \mathrm{~nm}$ there is a wider transmission range seen in both new and used sunglasses; although $84 \%(32 / 38)$ had a measured spectral transmittance of less than $2 \%$ at $400 \mathrm{~nm}$. The sunglasses with the highest spectral transmittance at $400 \mathrm{~nm}$ were a pair of used sunglasses at $7.0 \%$.

There was more variation between sunglasses in the reduction of blue light exposure; however, there is no evidence that exposure during flight exceeds international guideline limits (24) for type II retinal photochemical damage. A pair of new sunglasses had the lowest (best) transmittance at $440 \mathrm{~nm}$ of $0.2 \%$. These sunglasses are specifically marketed for pilots and are advertised for their blue light blocking properties. All sunglasses measured would reduce the blue light exposure and would counter the mean increase in blue light at altitude. Therefore, the mean blue light exposure during flight with any sunglass type would be no greater than the equivalent un-protected ocular exposure at ground level.

Overall, there was no apparent difference in UV-A and blue light protection between used and new sunglasses measured; however, direct like for like comparison could be made only for a single sunglass model. Differences were minimal and transmittance at $380 \mathrm{~nm}$ was measured at $0.3 \%$ for both filters and $12.1 \%$ for the new filter and $12.5 \%$ for the used filter at $440 \mathrm{~nm}$. It is interesting to note that the sunglasses with the highest transmittance at $440 \mathrm{~nm}$ were used sunglasses and were also the only sunglasses to be recorded as having scratched or marked lenses. This finding is, perhaps, unsurprising as if the tint were applied to the lens surface during manufacture, subsequent surface damage could increase lens transmittance while UV-A attenuation is mainly attributed to the lens material and was not measurably affected by scratches. However, this finding is unlikely to be 
of practically significance unless significant scratching is present on the center of the sunglass filter in front of the pupil.

Spectral weighting values of $S(\lambda)$ function above $\sim 360 \mathrm{~nm}$ used to calculate UV-A transmittance specified by the ISO 12311 Standard are very low: $1.3 \times 10^{-4}$ at $360 \mathrm{~nm}$, decreasing to $6.4 \times 10^{-5}$ at $380 \mathrm{~nm}$ and $3 \times 10^{-5}$ at $400 \mathrm{~nm}$. Therefore, the weighted UV-A transmittance of a filter is largely unaffected by transmission in the $360-400 \mathrm{~nm}$ spectral range and changing UV-A boundaries from $380 \mathrm{~nm}$ to $400 \mathrm{~nm}$ would have negligible effect on weighted transmittance. A sunglasses filter which has significant transmission in the range $360-400 \mathrm{~nm}$ would still be well within the category 2 and 3 UV-A limits when spectral weighting is applied. Although no such filters were discovered in this study, it would potentially produce an ISO compliant filter which could result in an increased UV-A exposure to a pilot. This also offers explanation as to low numbers of UVR transmittance failures previously found (15) where a spectral weighting function is applied.

Both ISO 12311 and ANSI Z80.3 apply to non-prescription sunglasses and there is no requirement that prescription sunglasses are manufactured to meet the same standards; this study showed no evidence to suggest that the non-prescription sunglasses measured do not meet ISO or ANSI UVR transmittance requirements. Prescription lenses are often dyed in a tint bath to the desired color and depth in an optical glazing laboratory. This will affect the luminous transmittance of the lens but does not automatically guarantee good UV-A protection unless a lens material which offers good UV-A attenuation is used or a UVR blocking coating is applied. It is known that a significant proportion of professional pilots are required to use optical correction in flight (9). Therefore for illustration, table II shows an example of two pairs of used pilot prescription sunglasses compared to the middle section of a typical non-prescription graduated tint filter. Although the possibility of beam refraction through a prescription lens is recognised as a potential source of discrepancies in transmission measured by different methods (34), it has also been previously reported that the beam refraction effect is not significant (19). While weighted UV-A transmittance are similar, 
average UV-A (calculated according to ANSI Z80.3:2010 or as average in $315-400 \mathrm{~nm}$ ) and average $380-400 \mathrm{~nm}$ transmittance are significantly different for the two pairs of prescription sunglasses. It is postulated that the lenses in prescription sunglass 1 were purely dyed without enhanced UVR protection while prescription sunglass 2 was manufactured to have superior UVR protection.

[Table II here]

In order to ensure protection from UVR induced cataract, ICNIRP recommended that the effective radiant exposure for wavelengths between 315 to $400 \mathrm{~nm}$ to the unprotected eye should not exceed $1 \times 10^{4} \mathrm{Jm}^{-2}$ within an 8 hour period. ICNIRP state that the exposure limits should be considered an absolute for direct exposure of the eye (21); this recommendation is supported by WHO and ILO (22). Combination of very high UV-A irradiance in 380-400nm at flight altitude, insufficient attenuation by sunglasses in this spectral region and a degree of pupil dilation behind a sunglass filter might underestimate the risk of ocular exposure (19).

Fig. 2 illustrates this on an example of cockpit irradiance data measured behind the front windshield in the study reported by Chorley et al (10) and the calculated subsequent irradiance reaching the eye using one of the three pairs of sunglasses shown in Table II; spectral irradiance is given on logarithmic scale. Radiant UV-A exposure for unprotected eyes during this single flight was $\sim 6.5$ times above the ICNIRP guidance; this value may be higher during different seasons and/or on flights to other destinations. Prescription sunglass 1 would provide a reduction in ocular UV-A exposure by a factor of $\sim 7.6$ whilst the graduated tint sunglass shown in this table would reduce ocular UV-A exposure by nearly a factor of 2000. It should be noted that UV-A transmittance of these three pairs of sunglasses complies with requirements of both ISO and ANSI standards; however, the reduction of UV-A in-flight afforded by prescription sunglass 1 may not be sufficient.

[Fig. 2. Here] 
Based on the data presented above, it is suggested that in order to guarantee adequate UVR protection for pilots during flight, compliance with the requirements of ISO 12311 or ANSI Z80.3:2010 should be combined with a $10 \%$ upper limit of spectral transmittance at $380 \mathrm{~nm}$. This would ensure that the filter would sufficiently attenuate the high intensity longer waveband UV-A which professional pilots are subject to (10) so that ocular exposure would not exceed international guideline limits (21). This proposed requirement was met by all new and used non-prescription sunglasses assessed in this study.

A number of materials are used in the manufacture of spectacle lenses and sunglass filters including CR39, polycarbonate, crown glass and Trivex. Some materials, such as Trivex, have inherently greater UV-A blocking properties although sunglass filters may be made from other materials and appropriately treated during manufacture to ensure the filter's compliance with solar UVR blocking requirements. It should be emphasized that UVR blocking dopants or UVR blocking filters do not compromise visible light transmission.

There are criteria for sunglasses other than ocular protection from solar radiation. For example, sunglasses should not impair pilots' color perception and should be comfortable and compatible with a headset. The sunglass frame should be of sufficient size to ensure protection from peripheral radiation and be thin so that a significant visual field artefact is not induced. Finally, a graduated tint may be helpful to pilots when viewing aircraft instrument displays. Provided there is good UVR attenuation of the lens material, the evidence from this study indicates that the lower portion of a graduated lens still comfortably provides adequate UVR protection during flight as transmittance from the lower portion of all graduated filters measured was within $0.6 \%$ at $380 \mathrm{~nm}$ and $6.1 \%$ at $400 \mathrm{~nm}$.

Effectiveness of UVR and blue light attenuation of the typical sunglasses used by pilots was analysed based on measurements of spectral transmittance and in-flight exposure data. All non-prescription 
uniform and graduated tints tested showed very high UVR attenuation. However, analysis of the data from prescription sunglasses raised concern that some of these may not provide sufficient ocular UVR protection during flight. It is suggested that to ensure adequate protection of pilots during flights, the requirements of international standard ISO 12312-1 or ANSI Z80.3:2010 should be combined with a $10 \%$ upper limit of spectral transmittance at $380 \mathrm{~nm}$. Additionally, pilots using prescription sunglasses should ensure that the lenses are either made from a material which effectively blocks UVR or have a UVR blocking coating. Sunglass lenses should be clean and to ensure continued optimum protection, replaced when significant scratches to the lens surface are present. 


\section{References}

1. Algvere PV, Marshall J, Seregard S. Age-related maculopathy and the impact of blue light hazard. Acta Ophthalmol Scand. 2006; 84(1):4-15.

2. ANSI Z80.3-2010 American National Standards for Ophthalmics - Nonprescription Sunglass and Fashion Eyewear Requirements. Alexandria (VA): The Vision Council; 2010.

3. Australian/New Zealand Standard. AS/NZS 1067:2003. Sunglasses and fashion spectacles. Syndey: Standards Australia; 2003.

4. Blumthaler M, Ambach W, Ellinger R. Increase in solar UV radiation with altitude. J Photochem Photobiol B. 1997; 39(2):130-134.

5. Brainard GC, Hanifin JP, Greeson JM, Byrne B, Glickman G, Gerner E, Rollag MD. Action Spectrum for Melatonin Regulation in Humans: Evidence for a Novel Circadian Photoreceptor. J Neurosci. 2001; 21(16):6405-6412.

6. Chorley AC, Evans BJW, Benwell MJ. Civilian pilot exposure to ultraviolet and blue light and pilot use of sunglasses. Aviat Space Environ Med. 2011; 82(9):895-900.

7. Chorley AC, Evans BJW, Benwell MJ. Solar eye protection habits of civilian professional pilots. Med Aeronaut Spat. 2013; 54(202):61-67.

8. Chorley AC, Higlett M, Baczynska K, Hunter R, Khazova M. Measurements of Pilots' Occupational Solar UV Exposure. Photochem Photobiol. 2014; 90(4):935-940.

9. Chorley AC, Evans BJW, Benwell MJ. Solar eye protection practices of civilian aircrew. Aerosp Med Hum Perform. 2015; 86(11):1 - 9. 
10. Chorley AC, Baczynska K, Benwell MJ, Evans BJW, et al. Occupational ocular UV exposure in civilian aircrew. Aerosp Med Hum Perform. Forthcoming.

11. Civil Aviation Authority. Spectacle frame and lens choice. Civil Aviation Authority. [Accessed 2015 Sept 18] Available from: http://www.caa.co.uk/default.aspx?catid=49\&pagetype=90\&pageid=9247

12. Cruickshanks KJ, Klein BE, Klein R. Ultraviolet light exposure and lens opacities: the Beaver Dam Eye Study. Am J Public Health. 1992; 82(12):1658-1662.

13. Cruickshanks KJ, Klein R, Klein BE. Sunlight and age-related macular degeneration. The Beaver Dam Eye Study. Arch Ophthalmol. 1993; 111(4):514-518.

14. Dain SJ. Comparison of the transmittance and coloration requirements of the four national sunglass standards. Optom Vis Sci. 1993; 70(1):66-74.

15. Dain SJ, Ngo TP, Cheng BB, Hu A, Teh AG, Tseng J, Vu N. Sunglasses, the European directive and the European standard. Ophthalmic Physiol Optics. 2010; 30(3):253-256.

16. Delcourt C, Cristol JP, Tessier F, Leger CL, Michel F, Papoz L. Risk factors for cortical, nuclear, and posterior subcapsular cataracts: the POLA study. Pathologies Oculaires Liees a I'Age. Am J Epidemiol. 2000; 151(5):497-504.

17. Diffey BL, Roscoe AH. (1990) Exposure to solar ultraviolet radiation in flight. Aviat Space Environ Med. 1990; 61(11):1032-1035.

18. Federal Aviation Administration. Sunglasses for pilots: Beyond the image. FAA [Accessed 2015 Sept 19]. Available from:

http://www.faa.gov/pilots/safety/pilotsafetybrochures/media/sunglasses.pdf

19. Gies P, Roy CR. Ocular Protection from ultraviolet radiation. Clin Exp Optom. 1988; 71(1):21-27. 
20. Ham WJ Jr, Mueller HA. The Photopathology and Nature of the Blue Light and Near-UV Retinal Lesions Produecd by Lasers and Other Optical Sources, In Wolbarsht, M.L. (ed.) Laser Applications in Medicine and Biology. New York: Plenum Publishing Corp; 1989. pp. 191-246.

21. International Commission on Non-ionising Radiation Protection. ICNIRP guidelines on limits of exposure to ultraviolet radiation of wavelengths between $180 \mathrm{~nm}$ and $400 \mathrm{~nm}$ (incoherent optical radiation). Health Phys. 2004; 87(2):171-186.

22. International Commission on Non-Ionizing Radiation Protection. Protecting workers from ultraviolet radiation. ICNIRP/WHO/ILO. Oberschleissheim: ICNIRP; 2007.

23. International Commission on Non-Ionizing Radiation Protection Statement. On Protection of workers against ultraviolet radiation. Health Phys. 2010; 99(1):66-87.

24. International Commission on Non-ionising Radiation Protection. ICNIRP guidelines on limits of exposure to incoherent visible and infrared radiation. Health Phys. 2013; 105(1):74-96.

25. International Standard. ISO 11664-2. Colorimetry -- Part 2: CIE standard illuminants. Switzerland: ISO; 2007.

26. International Standard. ISO 12312-1. Eye and face protection - Sunglasses and related eyewear Part 1: Sunglasses for general use. Switzerland: ISO; 2013.

27. International Standard. ISO 12311:2013, Corrected version 2014-08-15. Personal protective equipment - Test methods for sunglasses and related eyewear. Switzerland: ISO; 2013.

28. International Standard ISO 21348. Space environment (natural and artificial) - Process for determining solar irradiances. Switzerland: ISO; 2007.

29. Kuyk TK, Thomas SR. Effect of short wavelength absorbing filters on Farnsworth-Munsell 100 Hue test and hue identification task performance. Optom Vis Sci. 1990; 67(7): 522-531. 
30. McCarty CA, Nanjan MB, Taylor HR. Attributable risk estimates for cataract to prioritize medical and public health action. Invest Ophthalmol Vis Sci. 2000; 41(12):3720-3725.

31. Nakagawara VB, Montgomery RW, Marshall WJ. Optical Radiation Transmittance of Aircraft Windscreens and Pilot Vision. Oklahoma City, OK: Federal Aviation Administration Civil Aerospace Medical Inst; 2007.

32. Oliva MS, Taylor H. Ultraviolet radiation and the eye. Int ophthalmol clin. 2005; 45(1):1-17.

33. Sanlorenzo M, Wehner MR, Linos E, Kornak J, Kainz W, Posch C, et al. The Risk of Melanoma in Airline Pilots and Cabin Crew: A Meta-analysis. JAMA Dermatol. 2014; 151(1):51-58.

34. Stephens GL, Pitts DG. Evaluation of the performance of spectacle lens "Transmittance Meters". Optom Vis Sci. 1994; 71(3)220-225.

35. Taylor HR, West S, Munoz B, Rosenthal FS, Bressler SB, Bressler NM. The long-term effects of visible light on the eye. Arch Ophthalmol. 1992; 110(1):99-104.

36. Wang JJ, Jakobsen K, Smith W, Mitchell P. Five-year incidence of age-related maculopathy in relation to iris, skin or hair colour, and skin sun sensitivity: the Blue Mountains Eye Study. Clin Experiment Ophthalmol. 2003; 31(4):317-321.

37. Wang SQ, Setlow R, Berwick M, Polsky D, Marghoob AA, et al. Ultraviolet A and melanoma: a review. J Am Acad Dermatol. 2001; 44( 5): 837 - 846.

38. World Health Organization . Th e eff ects of Solar UV Radiation on the eye. WHO/PBL/EHG/94.1. Geneva: World Health Organisation ; 1993.

39. Young RW. Solar radiation and age-related macular degeneration. Surv Ophthalmol. 1988; $32(4): 252-269$. 


\begin{tabular}{|c|c|c|c|c|}
\hline \multirow[b]{2}{*}{ Make } & \multicolumn{4}{|c|}{ Filter tint type } \\
\hline & uniform & ted & rm & ated new \\
\hline RayBan & 5 & 5 & 1 & 2 \\
\hline Oakley & 5 & 0 & 4 & 0 \\
\hline Prada & 1 & 0 & 1 & 2 \\
\hline Police & 2 & 0 & 2 & 1 \\
\hline Ted Baker & 1 & 0 & 0 & 0 \\
\hline Lacoste & 0 & 0 & 0 & 1 \\
\hline Marks \& Spencer & 1 & 0 & 0 & 0 \\
\hline Caruso \& Freeland & 0 & 0 & 1 & 0 \\
\hline Bigatmo & 0 & 0 & 3 & 0 \\
\hline Total & 15 & 5 & 12 & 6 \\
\hline
\end{tabular}

Table I. new and used sunglasses measured 


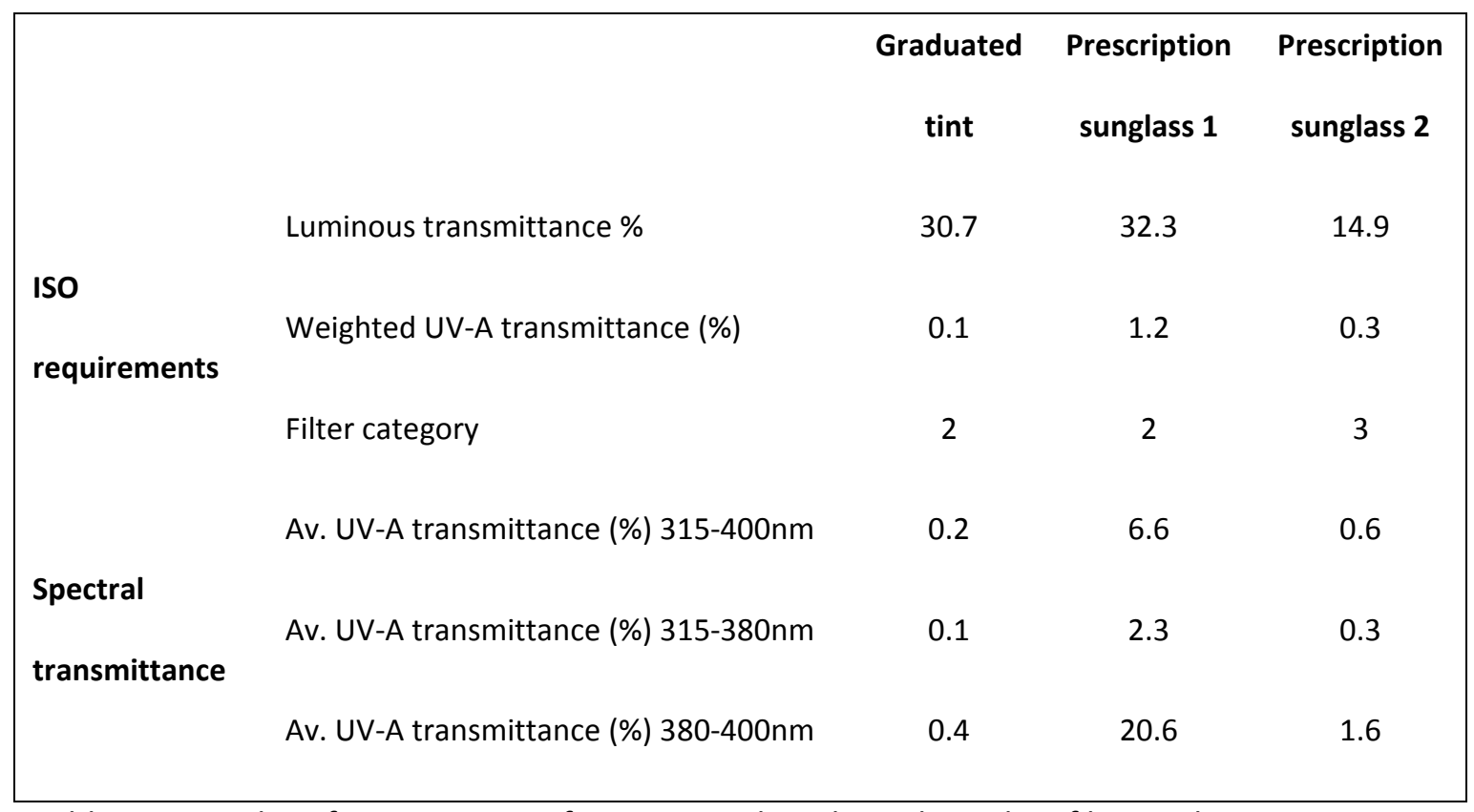

Table II. Examples of transmittance from a typical graduated sunglass filter and two prescription sunglasses. 
Fig. 1. Maximum UV-A transmittance of uniform and graduated tint sunglasses for used and new pairs. Each group is shown as a percentage of measured samples in each category, uniform or graduated.

Fig. 2. Spectral irradiance in cockpit during flight and spectral irradiance attenuated by sunglasses shown in Table II. 